\title{
Risk factors for progression toward brain death after out-of-hospital cardiac arrest
}

\author{
Martin Cour ${ }^{1,2,3}$, Jean Turc ${ }^{1}$, Thomas Madelaine ${ }^{1,2}$ and Laurent Argaud ${ }^{1,2,3^{*}}$ (1)
}

\begin{abstract}
Background: Successfully resuscitated out-of-hospital cardiac arrest (OHCA) may lead to brain death (BD) and goodquality transplantable organs. We aimed to determine risk factors for evolution toward BD after OHCA. We analyzed adult patients admitted to an intensive care unit (ICU) who survived at least $24 \mathrm{~h}$ after an OHCA between 2005 and 2015. BD was defined according to international guidelines. Multivariate logistic regression was used to identify potential risk factors for $\mathrm{BD}$ available $24 \mathrm{~h}$ after OHCA.

Results: A total of 214 patients were included (median age 68 years; sex ratio 1.25; non-shockable OHCA: 88\%). Among these, 42 (19.6\%) developed BD, while 22 (10.3\%) were alive at 1 year with a good neurological outcome. Independent risk factors for BD were age (OR per year 0.95; 95\% Cl [0.92-0.98]), female gender (OR 2.34; $95 \% \mathrm{Cl}$ [1.02-5.35]), neurological cause of OHCA (OR 14.72; 95\% Cl [3.03-71.37]), duration of the low-flow period > 16 min (OR $2.94,95 \% \mathrm{Cl}[1.21-7.16])$ and need of vasoactive drugs at $24 \mathrm{~h}$ (OR 6.20, 95\% Cl [2.41-15.93]).

Conclusions: The study identified, in a population of OHCA with predominantly non-shockable initial rhythms, five simple risk factors independently associated with progression toward BD.
\end{abstract}

Keywords: Cardiac arrest, Resuscitation, Brain death, Organ donation, Prognostication, Post-cardiac arrest syndrome

\section{Background}

Each year more than 300,000 patients experience an out-of-hospital cardiac arrest (OHCA) in both Europe and the USA [1, 2]. Even in patients in whom successful return of spontaneous circulation (ROSC) has been obtained, prognosis remains very poor [2]. This is particularly true for the increasing number of patients resuscitated by the emergency medical system presenting with non-shockable cardiac arrest rhythms (i.e., pulseless electrical activity and asystole), now accounting for approximately three-quarters of OHCAs $[1,2]$. Overall, $<10 \%$ of the patients admitted to intensive care unit (ICU) after OHCA are discharged without significant neurological sequelae $[1,2]$.

Great efforts have been made to develop tools to identify the patients who will most benefit from intensive

\footnotetext{
${ }^{*}$ Correspondence: laurent.argaud@chu-lyon.fr

${ }^{1}$ Hospices Civils de Lyon, Hôpital Edouard Herriot, Service de Médecine Intensive-Réanimation, 5, Place d'Arsonval, 69437 Lyon Cedex 03, France Full list of author information is available at the end of the article
}

care [3]. Several scores, biomarkers, and/or neurological exams may help clinicians to avoid futile care by predicting poor outcomes early after ICU admission [3]. However, the use of costly and time-consuming invasive treatments may have to be considered for some patients with dismal prognosis in order to create opportunities for organs donation. This is illustrated by several cohorts that indicate that brain death (BD) may occur in a sixth to a half of successfully resuscitated patients [4-9]. Moreover, it is now established that the quality of organ from OHCA brain-dead donors is similar to that of brain-dead patients from other causes $[5,9,10]$.

Predicting the occurrence of $\mathrm{BD}$ in comatose survivors of OHCA remains an unsolved challenge. Nevertheless, it would be useful to prognosticate BD after ICU admission. To date, only one study sought to do so but failed to determine risk factors for $\mathrm{BD}$ after OHCA among clini$\mathrm{cal}$ and biological variables available at the time of ICU admission [5]. We hypothesized that data available at $24 \mathrm{~h}$ after ICU admission from patients alive at this point 
in time would be more likely to identify risk factors for BD.

\section{Methods Study design}

We analyzed all consecutive OHCA patients admitted to a 15-bed university-affiliated medical ICU from 2005 to 2015. The study received approval from the local ethics committee (Comité de Protection des Personnes Sud-Est II). This institutional review board waived the need for consent given the retrospective and non-interventional design of the project.

\section{Data collection}

During the study period, all patients aged over 18 years admitted to the ICU after a non-traumatic OHCA and alive at $24 \mathrm{~h}$ after ICU admission (Day 1) were included. Data regarding OHCA and cardiopulmonary resuscitation were characterized according to Utstein Style [11]. Given the possible association with a high risk of BD [7, $12,13]$, the number of patients with neurological cause of cardiac arrest (stroke and status epilepticus) or hanging was also reported. The following information was also recorded at Day 1: hemodynamics and use of vasoactive drugs, temperature and targeted temperature management (TTM), continuous sedation, neurological evaluation, laboratory test data (most pejorative values during the first $24 \mathrm{~h}$ in ICU), Sequential Organ Failure Assessment (SOFA) score [14], and Simplified Acute Physiology Score II (SAPS II) [15].

Survival and neurological outcomes (Glasgow-Pittsburgh Cerebral Performance Categories, CPC [11]) were assessed at Day 90. Good neurological outcome was defined as a CPC 1 or 2. BD was defined according to international guidelines [16]. As recommended, the clinical diagnosis of $\mathrm{BD}$ was made in the absence of confounding factors (e.g., residual sedation) [16]. Ancillary tests (e.g., computed tomography angiograph or electroencephalogram) were used to confirm BD when the apnea test could not be performed and/or, according to French law, when organ donation was considered [17].

In case of $\mathrm{BD}$, data collected referred to the occurrence of organ procurement, the presence of a contraindication to organ donation, refusal, or other reasons for non-procurement.

\section{Post-cardiac arrest care}

During the study period, all patients admitted to our ICU after OHCA were managed according to international guidelines for post-cardiac arrest care [18, 19]. TTM was considered for all patients, for a period of 12-24 h, with a target temperature of $32-34{ }^{\circ} \mathrm{C}$ (external cooling). Midazolam and sufentanil were given as a continuous sedation. Sedation and analgesia protocols were based on the Richmond Agitation-Sedation Scale (RASS) and the behavioral pain scale, respectively [20]. Neuromuscular blocking agents could be used to prevent shivering during TTM. As recommended, we aimed to maintain the essential biological variables within physiological limits. There was no significant change in our protocol for postcardiac arrest care during the study period.

\section{Statistical analysis}

Values are presented as median and interquartile range (IQR) or number and percentage (\%), as appropriate. Univariate comparisons were performed using MannWhitney $U$ test for continuous variables, and Chisquare or Fisher's exact test for categorical variables, as appropriate.

Backward stepwise multivariate analysis using a logistic regression model was performed to assess the factors predicting evolvement toward BD. Following univariate analysis, variables with $p \leq 0.10$ and/or clinical pertinent variables were included in the model. Given uncertainty about the duration of no flow in patients with unwitnessed OHCA, these data were not entered in the model. Because of nonlinearity, the "low-flow period" data were transformed into a dummy variable according to its median value. Data which were part of the definition of BD were not included in the model. Because of the collinearity with SAPS II and/or the use of vasoactive drugs, SOFA score, epinephrine doses, mean arterial pressure, heart rate, and biological variables linked with organ dysfunctions were also not included in the model as stand-alone variables. Potential confounding factors were eliminated if $p$ value was $>0.10$. Odds ratios (OR) were estimated with $95 \%$ confidence intervals (95\% CI). Discrimination of the model was evaluated by measuring the area under the receiver operating characteristics (ROC) curve. MedCalc Statistical Software version 12.1.2 for windows (MedCalc Software BVBA, Ostend, Belgium) was used for all analyses. The significance level was set at $p<0.05$.

\section{Results}

During the study period, there were 304 patients admitted to ICU after OHCA; 214 of whom were alive at Day 1 and were included in the study (Fig. 1). Among these, $22(10 \%)$ had good neurological outcomes, whereas 42 (20\%) evolved toward BD (Fig. 1). Clinical BD diagnosis was confirmed by an ancillary test in the vast majority of cases $(n=36 / 42,86 \%)$. The median time elapsed between ICU admission and BD diagnosis was $2(2-3)$ days. A total of 23 organs procured from 7 brain-dead patients were successfully transplanted ( 3 hearts, 6 livers, and 14 kidneys). In the other 35 brain-dead patients, the main 


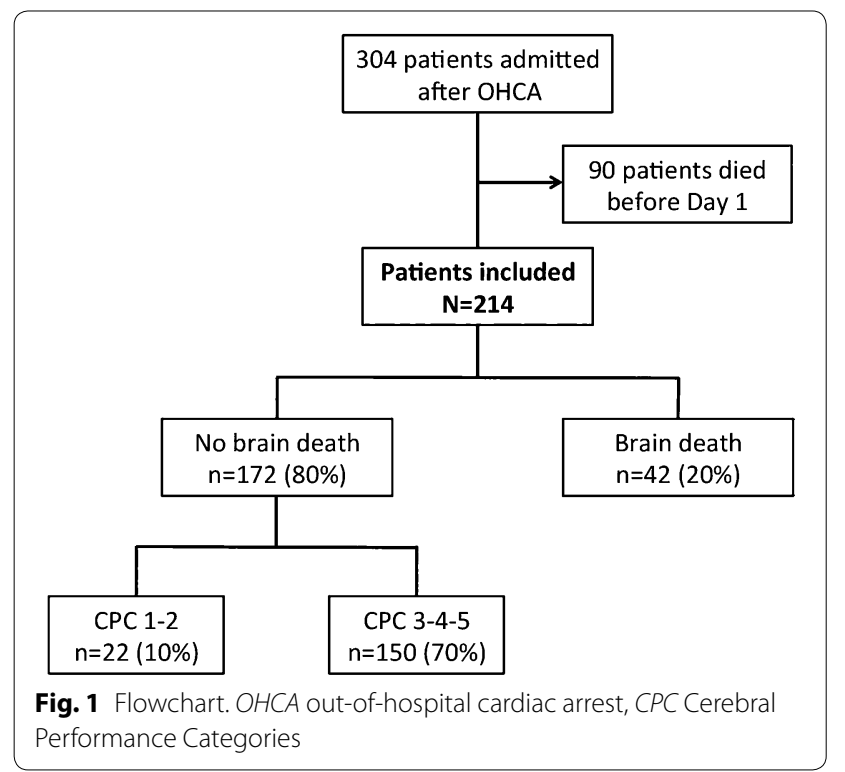

reasons for non-procurement were next-of-kin refusal $(\mathrm{n}=14)$ and contraindication related to multiple organ failure $(\mathrm{n}=11)$.

The baseline characteristics of the patients according to $\mathrm{BD}$ are presented in Table 1 . The majority of patients presented with OHCA of non-cardiac origin and nonshockable rhythms (Table 1). The median length of stay in ICU was 4 (2-6) days. In the no BD group, a high proportion of deaths occurred after withdrawal of lifesustaining therapy $(\mathrm{n}=102 / 136,75 \%)$ with a median delay from ICU admission of 5 (4-7) days. The age, the proportion of shockable rhythm, the proportion of witnessed OHCA, as well as resuscitation data significantly differed between patients with and without BD (Table 1). Patients with BD had a neurological cause of OHCA in 7 $(17 \%)$ cases (stroke $\mathrm{n}=5$, and status epilepticus $\mathrm{n}=2$ ) as compared to $5 / 172(3 \%)$ in the group with no BD (stroke $\mathrm{n}=4$ and status epilepticus $\mathrm{n}=1)(p<0.01)$. BD occurred in $7 / 15(47 \%)$ patients following hanging. As shown in

Table 1 Patient and cardiac arrest characteristics

\begin{tabular}{|c|c|c|c|c|}
\hline & All patients $(n=214)$ & $\begin{array}{l}\text { No brain death } \\
(n=172)\end{array}$ & Brain death $(n=42)$ & $p$ \\
\hline Age, years & $68(57-77)$ & $70(60-78)$ & $58(45-70)$ & $<0.001$ \\
\hline Male gender & $119(56)$ & $101(59)$ & $18(43)$ & 0.08 \\
\hline \multicolumn{5}{|l|}{ Comorbidities } \\
\hline Hypertension & $85(40)$ & $70(41)$ & $15(36)$ & 0.60 \\
\hline COPD & $58(27)$ & $45(26)$ & $13(31)$ & 0.56 \\
\hline Congestive heart failure & $33(15)$ & $27(16)$ & $6(14)$ & $>0.99$ \\
\hline Diabetes & $48(22)$ & $43(25)$ & $5(12)$ & 0.09 \\
\hline Chronic renal failure & $12(6)$ & $10(6)$ & $2(5)$ & 0.17 \\
\hline Presumed cause of cardiac arrest & & & & 0.30 \\
\hline Cardiac & $44(21)$ & $39(23)$ & $5(12)$ & \\
\hline Non-cardiac & $160(75)$ & $125(73)$ & $35(83)$ & \\
\hline Undetermined & $10(5)$ & $8(4)$ & $2(5)$ & \\
\hline Location of cardiac arrest & & & & 0.48 \\
\hline Home & $165(77)$ & $130(76)$ & $35(83)$ & - \\
\hline Public place & $45(21)$ & $39(23)$ & $6(14)$ & - \\
\hline Other & $4(2)$ & $3(2)$ & $1(2)$ & - \\
\hline Witnessed cardiac arrest & $166(78)$ & $139(81)$ & $27(64)$ & 0.02 \\
\hline First recorded cardiac rhythm & & & & 0.03 \\
\hline Shockable & $25(12)$ & $24(14)$ & $1(2)$ & - \\
\hline Non-shockable & $189(88)$ & $148(86)$ & $41(98)$ & - \\
\hline \multicolumn{5}{|l|}{ Resuscitation } \\
\hline Bystander CPR & $90(42)$ & $79(46)$ & $11(26)$ & 0.02 \\
\hline Time from collapse to ROSC, min & $25(15-35)$ & $22(15-32)$ & $32(23-46)$ & $<0.001$ \\
\hline No-flow period, min & $6(0-10)$ & $5(0-10)$ & $10(5-15)$ & $<0.001$ \\
\hline Low-flow period, $\min$ & $16(10-26)$ & $15(9-25)$ & $22(15-34)$ & $<0.001$ \\
\hline Dose of epinephrine, mg & $3(1-5)$ & $3(1-5)$ & $3(2-6)$ & 0.02 \\
\hline Number of defibrillation attempts & $0(0-1)$ & $0(0-1)$ & $0(0-1)$ & 0.76 \\
\hline
\end{tabular}

Data are expressed as median (interquartile range) or number (\%)

COPD chronic obstructive pulmonary disease, OHCA out-of-hospital cardiac arrest, $C P R$ cardiopulmonary resuscitation, ROSC restoration of spontaneous circulation 
Table 2, patients who evolved toward $\mathrm{BD}$ received more often vasoactive drugs for hemodynamic instability, had more often signs of brainstem dysfunction, and had more organ dysfunction at Day 1. In contrast, the occurrence of BD was significantly less frequent when patients still had pupillary light reflex or displayed myoclonus at Day 1 (Table 2).

Multivariate analysis identified 5 risk factors available at Day 1 , independently associated with BD after OHCA (Table 3). Neurological cause of cardiac arrest was the strongest predictor of $\mathrm{BD}$, followed by the need for vasoactive drugs at Day 1 (Table 3). The area under the ROC curve was 0.84 (95\% CI: 0.78-0.89).

\section{Discussion}

The present study provides the first insight into risk factors for developing BD after OHCA. Female gender, young age, neurological cause of cardiac arrest, duration of the low-flow period, and persistent hemodynamic shock were independently associated with the occurrence of BD. These five simple factors might help clinicians
Table 3 Independent predictors of brain death

\begin{tabular}{lccr}
\hline & Odds ratio & $\mathbf{9 5 \%} \mathbf{C l}$ & $\boldsymbol{p}$ \\
\hline Age, per year & 0.95 & $0.92-0.98$ & $<0.001$ \\
Female gender & 2.34 & $1.02-5.35$ & 0.043 \\
Neurological cause of cardiac arrest & 14.72 & $3.03-71.37$ & $<0.001$ \\
Duration of the low-flow & 2.94 & $1.21-7.16$ & 0.017 \\
$\quad$ period > 16 min & & & \\
Vasoactive drugs at Day 1 & 6.20 & $2.41-15.93$ & $<0.001$
\end{tabular}

$\mathrm{Cl}$ confidence intervals, $R O S C$ return of spontaneous circulation

to identify early a potential pool of future organ donors among OHCA patients.

In the present study, one in five patients who survived more than $24 \mathrm{~h}$ evolved toward BD. Moreover, 5 patients evolved to BD before Day 1 (data not shown). The high rate of $\mathrm{BD}$ following cardiac arrest has been known since the beginning of the 2000s [21]. The most recent systematic review including 23,388 patients indicates that $\mathrm{BD}$ might occur in about $10 \%$ of the OHCA [8]. However, this figure is probably underestimated as some patients with

\section{Table 2 Clinical and biological data at Day 1}

\begin{tabular}{|c|c|c|c|c|}
\hline & All patients $(n=214)$ & No brain death $(n=172)$ & Brain death $(n=42)$ & $p$ \\
\hline \multicolumn{5}{|l|}{ Hemodynamics } \\
\hline MAP, mmHg & $78(65-91)$ & $80(65-92)$ & $73(63-82)$ & 0.09 \\
\hline Heart rate, bpm & $100(85-114)$ & $98(82-112)$ & $110(95-119)$ & $<0.01$ \\
\hline Vasoactive drugs & $107(50)$ & $74(43)$ & $33(79)$ & $<0.01$ \\
\hline \multicolumn{5}{|l|}{ Temperature } \\
\hline Temperature, ${ }^{\circ} \mathrm{C}$ & $37.4(36.2-38.2)$ & $37.5(36.4-38.1)$ & $37.5(36.1-38.7)$ & 0.80 \\
\hline TTM & $102(48)$ & $81(47)$ & $21(50)$ & 0.86 \\
\hline Continuous sedation & $88(41)$ & $75(44)$ & $13(31)$ & 0.16 \\
\hline \multicolumn{5}{|l|}{ Neurological examination } \\
\hline GCS score & $3(3-4)$ & $3(3-5)$ & $3(3-3)$ & $<0.001$ \\
\hline GCS Motor subscore $\leq 2$ & $181(85)$ & $139(81)$ & $42(100)$ & $<0.001$ \\
\hline Myoclonus & $68(32)$ & $67(39)$ & $1(2)$ & $<0.001$ \\
\hline Pupillary light reflex present & $112(52)$ & $110(64)$ & $2(5)$ & $<0.001$ \\
\hline Spontaneous ventilation absent & $75(35)$ & $45(26)$ & $30(71)$ & $<0.001$ \\
\hline \multicolumn{5}{|l|}{ Biological variables } \\
\hline Arterial pH & $7.25(7.14-7.32)$ & $7.25(7.15-7.33)$ & $7.17(7.07-7.29)$ & 0.02 \\
\hline $\mathrm{PaCO}_{2}, \mathrm{KPa}$ & $5.3(4.5-6.5)$ & $5.3(4.6-6.5)$ & $5.4(4.4-7.0)$ & 0.97 \\
\hline Serum lactate, $\mathrm{mmol} / \mathrm{l}$ & $7.7(4.9-11.0)$ & $7.4(4.5-10.7)$ & $8.9(5.8-12.0)$ & 0.07 \\
\hline Troponin, $\mu \mathrm{g} / \mathrm{l}$ & $0.7(0.1-5.1)$ & $0.5(0.1-3.6)$ & $1,9(0.3-19.0)$ & $<0.01$ \\
\hline$A L T, I U / L$ & $104(44-261)$ & $84(39-206)$ & $178(92-376)$ & $<0.001$ \\
\hline Creatinine, $\mu \mathrm{mol} / \mathrm{I}$ & $110(81-173)$ & 107 (80-172) & $124(87-178)$ & 0.33 \\
\hline Platelets, $10^{9} /$ I & $201(155-256)$ & $208(157-261)$ & $181(144-242)$ & 0.29 \\
\hline SOFA score & $9(6-12)$ & $9(6-11)$ & $11(9-14)$ & $<0.001$ \\
\hline SAPS $\|$ & $74(65-86)$ & $75(64-86)$ & $74(69-86)$ & 0.91 \\
\hline
\end{tabular}

Data are expressed as median (interquartile range) or number (\%)

MAP mean arterial pressure, TTM targeted temperature management, GCS Glasgow Coma Scale, $P a C O_{2}$ arterial partial pressure of carbon dioxide, $A L T$ alanine aminotransferase, SOFA Sequential Organ Failure Assessment, SAPS // Simplified Acute Physiology Score II 
very poor prognosis might have died before BD diagnosis, due to intractable hemodynamic shock or early withdrawal of life support. Furthermore, one can reasonably assume that OHCA patients with the worst prognosis are often neither referred to centers of expertise nor enrolled in a study, which probably decreases their chance of being both diagnosed with $\mathrm{BD}$ and included in a cohort. In the aforementioned review, the authors also reported a wide range of BD incidence rates (from 0 to $43 \%$ ) suggesting that this event depends on the population studied. In the present study an overwhelming majority of patients had non-shockable OHCA, which are traditionally associated with dismal outcomes [1, 2, 22, 23]. As a result, the patients were twice more likely to develop BD than to survive without severe neurological sequelae. Thus, given the high proportion of $\mathrm{BD}$ after OHCA, when organ donation is an option, aggressive treatment appears to be appropriate. In this context, it would be helpful to have tools to prognosticate the occurrence of $\mathrm{BD}$, especially in patients with the poorest prognosis, in order to prevent futile care.

We identified five factors available at Day 1 independently associated with progression toward BD after OHCA. To the best of our knowledge, only one previous study, in which $16 \%$ of patients met criteria for BD, sought to do so [5]. Unfortunately, analyzing only data available at ICU admission, the authors failed to find independent early predictors of $\mathrm{BD}$. Consequently, we chose not to include patients who died within the first $24 \mathrm{~h}$ after ICU admission. Furthermore, BD prognostication is probably not relevant at a very early stage as most of deaths occurring within few hours after hospital admission result from intractable cardiovascular failure and multiple organ failure, contraindicating any organ procurement [14, 24]. Moreover, because of confounding factors such as sedation or hypothermia, BD diagnosis is barely feasible soon after admission [16]. Taking into accounts these considerations, we found that female gender, young age, neurological cause of cardiac arrest, duration of the low-flow period, and persistent hemodynamic shock, were independently associated with BD after OHCA. While one can reasonably assume that cerebral edema was more likely to induce BD in patients without brain atrophy (i.e., in young patients), the reasons why females had higher chance of BD remain to be determined. The other risk factors were related to the extent of the initial brain insult or the severity of the post-cardiac arrest syndrome.

These risk factors for BD can form the basis for a future simple score system. Providing a probability of BD after OHCA, such a score might help to adjust levels of care, especially when there is no chance of neurological recovery. The prospect of organ donation may also re-motivate the teams to optimize organ protection even in patients with the worse prognosis. Equally important, identifying patients at high risk of $\mathrm{BD}$ is primordial for information of families/relatives, notably regarding organ donation. Besides, given the fact that the delay between ICU admission and BD is relatively short after OHCA, physicians might also opt for short-acting sedation drugs and choose a target temperature that does not alter neuroelectrophysiologic tests.

We chose not to introduce neurological exam variables at Day 1 into the logistic regression model (i.e., GCS, pupillary reactivity, or myoclonus) because of redundancy with $\mathrm{BD}$ diagnosis criteria [16]. However, interestingly, some of these variables were strongly associated with the absence of subsequent occurrence of BD. For instance, none of the patients with a Glasgow motor subscore $>2$ at Day 1 developed BD. In the same way, patients with myoclonus and/or preserved pupillary light reflex at Day 1 , were very unlikely (up to $5 \%$ ) to progress to $\mathrm{BD}$. Taken together, these very simple clinical variables could contribute to identify at an early stage the patients who will not meet BD criteria after OHCA.

We acknowledge some limitations. First, given the retrospective analysis of a prospective basis, we might have omitted some confounders for the adjustment. Second, we did not analyze the predictive value of some prognostic factors such as early electroencephalogram or biomarkers of brain injury. Third, even though the discrimination of the prediction model was good, we cannot rule out the possibility of an overfitting related to the relatively low number of events per variable. Finally, our study only represents the characteristics of a single hospital and we performed statistical analysis with data from patients admitted mostly after non-shockable OHCA of non-cardiac origin. Therefore, even though BD was not independently associated with initial cardiac rhythm, further studies are needed to confirm our findings, especially in cohorts where OHCAs from cardiac origin predominate.

\section{Conclusions}

To the best of our knowledge, this study is the first to highlight demographic, anamnestic, and clinical variables independently associated with the occurrence of BD after OHCA. These findings might help physicians in their decision-making processes and could serve as a basis for developing a simple score that could accurately predict $\mathrm{BD}$ after OHCA.

\section{Abbreviations}

BD: brain death; CPC: Cerebral Performance Categories; Cl: confidence intervals; ICU: intensive care unit; OHCA: out-of-hospital cardiac arrest; OR: odds ratio; SOFA: Sequential Organ Failure Assessment. 


\section{Authors' contributions}

$M C$ and $L A$ conceived and designed the study and drafted the first version of the manuscript. MC, JT, and TM collected the data. MC, JT, TM, and LA analyzed the data. JT and TM critically revised the manuscript. All authors read and approved the final manuscript.

\section{Author details}

${ }^{1}$ Hospices Civils de Lyon, Hôpital Edouard Herriot, Service de Médecine Intensive-Réanimation, 5, Place d'Arsonval, 69437 Lyon Cedex 03, France. ${ }^{2}$ Faculté de médecine Lyon-Est, Université Claude Bernard Lyon 1, Université de Lyon, 69373 Lyon, France. ${ }^{3}$ U1060 CarMeN, INSERM, 69373 Lyon, France.

\section{Acknowledgements}

We thank Dr. Philip Robinson (DRCl, Hospices Civils de Lyon) for help in manuscript preparation.

\section{Competing interests}

The authors declare that they have no competing interests.

\section{Availability of data and materials}

A limited de-identified dataset is available from the corresponding author on reasonable request.

\section{Consent for publication}

Not applicable.

\section{Ethics approval and consent to participate}

The study received approval from the local ethics committee (Comite de Protection des Personnes Sud-Est II). This institutional review board waived the need for consent given the retrospective and non-interventional design of the project.

\section{Funding}

None.

\section{Publisher's Note}

Springer Nature remains neutral with regard to jurisdictional claims in published maps and institutional affiliations.

Received: 20 December 2018 Accepted: 2 April 2019 Published online: 08 April 2019

\section{References}

1. Mozaffarian D, Benjamin EJ, Go AS, et al. Heart disease and stroke statistics 2016 update: a report from the American Heart Association. Circulation. 2016;133:e38-360.

2. Berdowski J, Berg RA, Tijssen JG, Koster RW. Global incidences of out-ofhospital cardiac arrest and survival rates: systematic review of 67 prospective studies. Resuscitation. 2010;81:1479-87.

3. Rossetti A, Rabinstein AA, Oddo M. Neurological prognostication of outcome in patients in coma after cardiac arrest. Lancet Neurol. 2016;15:597-609.

4. Jouffroy R, Lamhaut L, Guyard A, et al. Early detection of brain death using the Bispectral Index (BIS) in patients treated by extracorporeal cardiopulmonary resuscitation (E-CPR) for refractory cardiac arrest. Resuscitation. 2017;120:8-13.

5. Adrie $\mathrm{C}$, Haouache $\mathrm{H}$, Saleh $\mathrm{M}$, et al. An underrecognized source of organ donors: patients with brain death after successfully resuscitated cardiac arrest. Intensive Care Med. 2008;34:132-7.
6. Faucher A, Savary D, Jund J, et al. Out-of-hospital traumatic cardiac arrest: an underrecognized source of organ donors. Transpl Int. 2014;27:42-8.

7. Arnaout M, Mongardon N, Deye N, et al. Out-of-hospital cardiac arrest from brain cause: epidemiology, clinical features, and outcome in a multicenter cohort. Crit Care Med. 2015:43:453-60.

8. Sandroni C, D'Arrigo S, Callaway CW, et al. The rate of brain death and organ donation in patients resuscitated from cardiac arrest: a systematic review and meta-analysis. Intensive Care Med. 2016;42:1661-71.

9. Sandroni C, Adrie C, Cavallaro F, et al. Are patients brain-dead after successful resuscitation from cardiac arrest suitable as organ donors? A systematic review. Resuscitation. 2010;81:1609-14.

10. Castelberry AW, Worni M, Osho AA, et al. Use of lung allografts from brain-dead donors after cardiopulmonary arrest and resuscitation. Am J Respir Crit Care Med. 2013;188:466-73.

11. Langhelle A, Nolan J, Herlitz J, et al. Utstein Consensus Symposium. Recommended guidelines for reviewing, reporting, and conducting research on post-resuscitation care. Resuscitation. 2005;66:271-83.

12. Wee $\mathrm{JH}$, Park KN, Oh SH, et al. Outcome analysis of cardiac arrest due to hanging injury. Am J Emerg Med. 2012;30:690-4.

13. Sutcliffe WJ, Saayman AG. Hanging and asphyxia: interventions, patient outcomes and resource utilisation in a UK tertiary intensive care unit. J Intensive Care Soc. 2018:19:201-8.

14. Cour M, Bresson D, Hernu R, et al. SOFA score to assess the severity of the post-cardiac arrest syndrome. Resuscitation. 2016;102:110-5.

15. LeGall JR, Lemeshow S, Saulnier F. A new Simplified Acute Physiology Score (SAPS II) based on a European/North American multicenter study. JAMA. 1993;270:2957-63.

16. Wijdicks EF, Varelas PN, Gronseth GS, Greer DM, American Academy of Neurology. Evidence-based guideline update: determining brain death in adults: report of the Quality Standard Subcommittee of the American Academy of Neurology. Neurology. 2010;74:1911-8.

17. Jousset N, Gaudin A, Mauillon D, et al. Organ donation in France: legislation, epidemiology and ethical comments. Med Sci Law. 2009;49:191-9.

18. ECC Committee, Subcommittees and Tasks Forces of the American Heart Association. Part 7.5: postresuscitation support: 2005 American Heart Association Guidelines for Cardiopulmonary Resuscitation and Emergency Cardiovascular Care. Circulation. 2005;2005(112):IV84-8.

19. Peberdy MA, Callaway CW, Neumar RW, et al. Part 9: post-cardiac arrest care: 2010 American Heart Association Guidelines for Cardiopulmonary Resuscitation and Emergency Cardiovascular Care. Circulation. 2011;124:e403.

20. Ely EW, Truman B, Shintani A, et al. Monitoring sedation status over time in ICU patients: reliability and validity of the Richmond Agitation-Sedation Scale (RASS). JAMA. 2003;289:2983-91.

21. Peberdy MA, Kaye W, Ornato JP, et al. Cardiopulmonary resuscitation of adults in the hospital: a report of 14720 cardiac arrests from the national registry of cardiopulmonary resuscitation. Resuscitation. 2003;58:297-308

22. Argaud L, Cour M, Dubien PY, et al. Effect of cyclosporine in non-shockable out-of-hospital cardiac arrest: the CYRUS randomized clinical trial. JAMA Cardiol. 2016;1:557-65.

23. Venet F, Cour M, Demarret J, et al. Decreased monocyte HLA-DR expression in patients after non-shockable out-of-hospital cardiac arrest. Shock. 2016;46:33-6.

24. Lemiale $\mathrm{V}$, Dumas $\mathrm{F}$, Mongardon $\mathrm{N}$, et al. Intensive care unit mortality after cardiac arrest: the relative contribution of shock and brain injury in a large cohort. Intensive Care Med. 2013;39:1972-80. 